\title{
Evaluation of the Bending Loss of the Hollow Optical Fiber for Application of the Carbon Dioxide Laser to Endoscopic Therapy
}

\author{
Daisuke Kusakari ${ }^{1}$, Hisanao Hazama ${ }^{1}$, Rinna Kawaguchi ${ }^{1}$, Katsunori Ishii ${ }^{1}$, Kunio Awazu ${ }^{1,2,3}$ \\ ${ }^{1}$ Graduate School of Engineering, Osaka University, Osaka, Japan \\ ${ }^{2}$ Graduate School of Frontier Biosciences, Osaka University, Osaka, Japan \\ ${ }^{3}$ The Center for Advanced Medical Engineering and Informatics, Osaka University, Osaka, Japan \\ Email: awazu@see.eng.osaka-u.ac.jp
}

Received April 29, 2013; revised June 30, 2013; accepted August 3, 2013

Copyright (C) 2013 Daisuke Kusakari et al. This is an open access article distributed under the Creative Commons Attribution License, which permits unrestricted use, distribution, and reproduction in any medium, provided the original work is properly cited.

\begin{abstract}
Since carbon dioxide laser is excellent for incision, hemostasis, coagulation, and vaporization of soft tissues, it has been widely applied in clinical treatments as the laser knife. In these days, flexible thin hollow optical fibers transmitting mid-infrared light have been developed, and the application of carbon dioxide laser to endoscopic therapy has become possible. However, it is expected that the irradiation effect is influenced by the change in the laser power at the tip of the hollow optical fiber due to the change in the transmittance by the bending loss. The purpose of this research is to quantitatively evaluate the change in the output power and therapeutic effect by bending the hollow optical fiber in a gastrointestinal endoscope. The change in the transmittance of the hollow optical fiber due to the insertion of the fiber into the endoscope and bending of the head of the endoscope was measured. Then, the relationship between the irradiated laser power and the incision depth for a porcine stomach was investigated. As the results, the most significant decrease in the transmittance of the hollow optical fiber was caused by the insertion of the fiber into the instrument channel of the endoscope, and bending of the head of the endoscope with the angle of $90^{\circ}$ decreased the output laser power and incision depth by $10 \%$ and $25 \%$, respectively. Therefore, it was confirmed that the bending loss of the hollow optical fiber due to the bending of the head of the endoscope had no significant influence on the endoscopic therapy using the carbon dioxide laser.
\end{abstract}

Keywords: Hollow Optical Fiber; Carbon Dioxide Laser; Bending Loss; Endoscopic Therapy

\section{Introduction}

Since carbon dioxide laser with the wavelength of 10.6 $\mu \mathrm{m}$ is absorbed strongly by water, which is contained about $70 \%$ in biological soft tissues, it is excellent in incision, hemostasis, coagulation, and vaporization of the soft tissues $[1,2]$. In fact, carbon dioxide laser is widely efficient for medical field, e.g., plastic surgery, cosmetic surgery, otolaryngology, and dentistry. Even in the endoscopic surgery, carbon dioxide laser had various promising application, so that many researchers developed new techniques for clinical treatment [3,4]. At the same time, several kinds of optical fibers transmitting midinfrared light have been developed. These optical fibers are classified broadly into three categories, i.e., glasses composed of fluoride or oxide, special glasses such as chalcogenide, and polycrystalline materials such as metal halide [5]. However, these optical fibers have problems in terms of the mechanical and chemical properties, toxicity, manufacturing cost, etc. Therefore, they could not be used for medical purposes. Recently, hollow optical fibers with a dielectric coating made of cyclic olefin polymer (COP) inside a cylindrical metal waveguide have been developed as shown in Figure 1 [6]. Since the core of the hollow optical fibers is air or other gases with a high transmittance at the wavelength of the carbon dioxide laser, extremely low absorption loss could be obtained [7]. Because the reflection loss at the end surface of the fiber and related damage do not occur, the hollow optical fibers are suitable to transmit high-power lasers. In addition, the hollow optical fiber shown in Figure 1 can simultaneously transmit carbon dioxide laser and visible aiming laser. Although it is possible to enhance the reflectance of the inner surface to nearly $100 \%$ by designing the COP layer thickness according to the wave- 
length of the transmitting laser light, the leaking loss to the outside of the fiber can not be eliminated in principle. Therefore, by bending the hollow optical fiber, the transmittance decreases due to the increase in the number of reflection per unit length and decrease in the reflectance in the fiber. When the hollow optical fiber is used in a clinical treatment with an endoscope, it is inferred that output laser power and therapeutic effect are changed due to the change in the transmittance of the hollow optical fiber caused by the bending of the endoscope. The purpose of this research is to quantitatively evaluate the change in the output laser power and therapeutic effect caused by bending the hollow optical fiber in a gastrointestinal endoscope.

\section{Materials and Methods}

\subsection{Transmittance of the Hollow Optical Fiber with Bending of the Endoscope}

In this research, a carbon dioxide laser system modified from a commercial laser system for dental treatment and so on (COM-2, J. Morita Manufacturing Corp., Kyoto, Japan) was used. The laser was operated in the continuous wave mode, and the maximum output power of the laser oscillator was $30 \mathrm{~W}$. Two types of the hollow optical fiber (J. Morita Manufacturing Corp.) listed in Table 1 were used to deliver the carbon dioxide laser through a gastrointestinal endoscope (GIF-2T200, Olympus Corp., Tokyo, Japan) shown in Figure 2. A laser power meter (30-A-BB-18, Ophir Optronics, Israel) was used to measure the laser power.

The carbon dioxide laser, the visible aiming laser with a wavelength of $650 \mathrm{~nm}$, and air were simultaneously transmitted through the hollow optical fiber. Input and output powers of the hollow optical fibers were measured under four different conditions as shown in Figure 3, and each condition was described as follows:

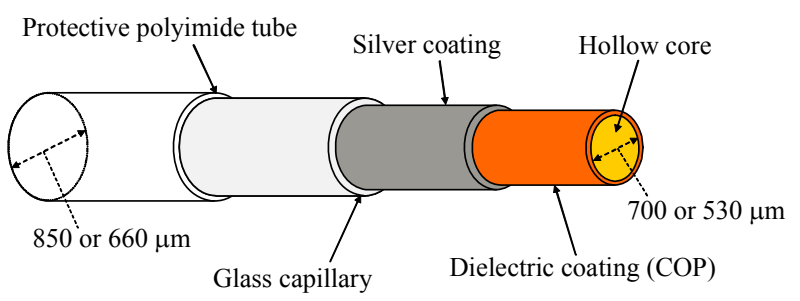

Figure 1. Schematic of the hollow optical fiber used in this research.

Table 1. Specifications of the hollow optical fibers.

\begin{tabular}{cccc}
\hline & Outer diameter $(\mu \mathrm{m})$ & Inner diameter $(\mu \mathrm{m})$ & Length $l(\mathrm{~cm})$ \\
\hline L-1 & 850 & 700 & 250 \\
L-2 & 850 & 700 & 260 \\
S-1 & 660 & 530 & 260 \\
\hline
\end{tabular}

I. The hollow optical fiber was kept straight without insertion to the endoscope.

II. The hollow optical fiber was inserted into the endoscope, and endoscope was kept straight.

III. In addition to the condition II, the middle part of the endoscope was gradually bent $90^{\circ}$ with a radius $r_{1}=$ $50 \mathrm{~cm}$.

IV.In addition to the condition III, the head of the endoscope was steeply bent $90^{\circ}$ with a radius $r_{2}=5 \mathrm{~cm}$.

\subsection{Dependence of the Output Laser Power and Incision Depth on the Bending of the Head of the Endoscope}

By assuming the case where the endoscope is used in transoral surgery, the middle part of the endoscope was bent $90^{\circ}$ with a radius $r_{1}=50 \mathrm{~cm}$, and the tip of the hollow optical fiber L-1 was bent with various angles and radii $r_{2}$ as listed in Table 2 . To avoid the accidental damage to the endoscope due to the break of the hollow optical fiber during laser irradiation, the fiber was ejected about $15 \mathrm{~cm}$ from the head of the endoscope, and only the fiber was bent by using in-house guide plates made of foamed polystyrene in order to simulate the bending conditions of the head of the endoscope.

A segment of a porcine stomach was set on a motorized linear stage (SGSP20-20, SIGMA KOKI Co., Ltd., Tokyo, Japan) and moved at a constant speed of 1.0 $\mathrm{mm} / \mathrm{s}$ during laser irradiation for a time of $20 \mathrm{~s}$. The car-

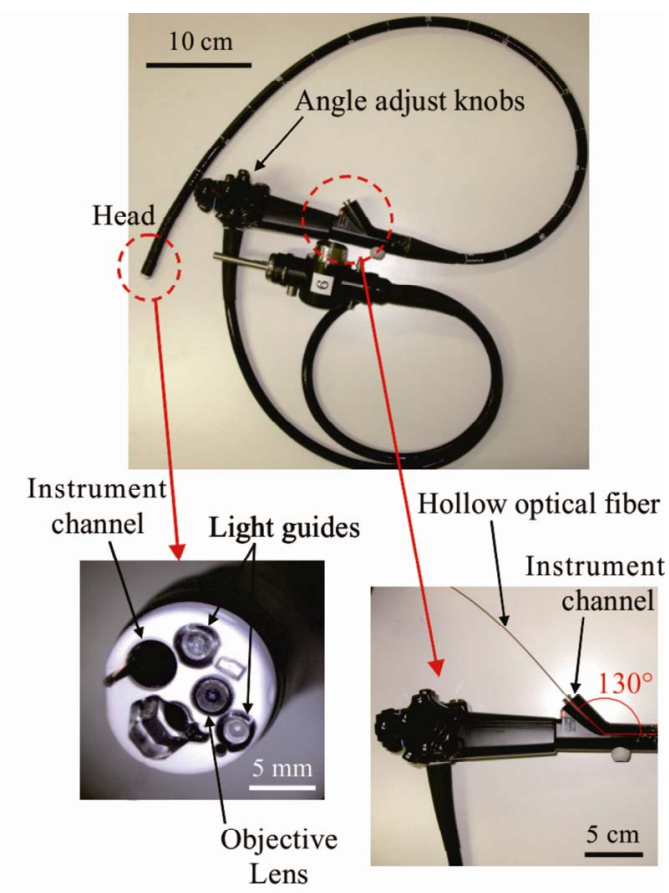

Figure 2. The photographs of the endoscope and its head and instrument channel. The hollow optical fiber is steeply bent $130^{\circ}$ just after the insertion to the instrument channel. 


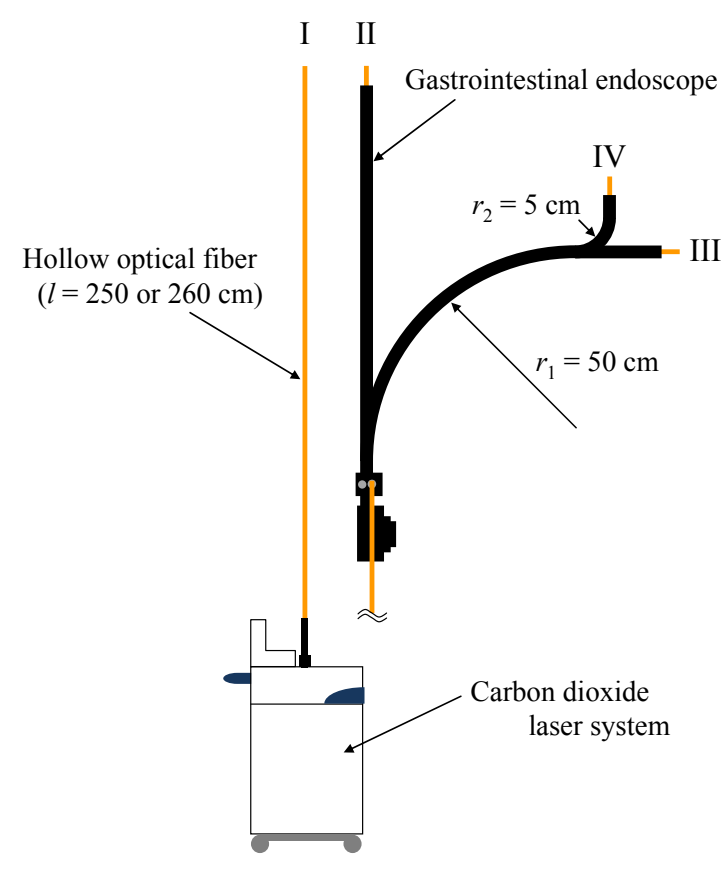

Figure 3. Schematics of the measurement of the transmittance of the hollow optical fibers for various bending conditions.

bon dioxide laser was irradiated vertically to the surface of the mucosa from a distance of $2 \mathrm{~mm}$. The setting power of the carbon dioxide laser system was set at 3,5 , and $8 \mathrm{~W}$, and laser power transmitted through the hollow optical fiber was measured under each condition. To simulate the in vivo environment, the sample surface was kept wet by pouring saline on the sample at a rate of 120 $\mathrm{mL} / \mathrm{h}$ using a micro syringe pump (IC3100, AS ONE, Osaka, Japan). After laser irradiation, each sample was stored at $-80^{\circ} \mathrm{C}$ and was sliced to a thickness of $10 \mu \mathrm{m}$ by using a cryostat microtome (CM1850, Leica Microsystems, Wetzlar, Germany) at a temperature of $-20^{\circ} \mathrm{C}$. Then, each sample section attached onto a glass slide was stained with hematoxylin and eosin (HE) staining and observed using a high-resolution slide scanner (NanoZoomer 2.0 RS, Hamamatsu Photonics K. K., Shizuoka, Japan).

\section{Results and Discussion}

\subsection{Transmittance of the Hollow Optical Fiber with Bending of the Endoscope}

Figure 4 shows the transmittance of the hollow optical fibers under each condition. When the hollow optical fiber was inserted into the instrument channel of the endoscope (from the condition I to II), the transmittance of the fiber L-1, L-2, and S-1 decreased by $29 \%, 28 \%$, and $24 \%$, respectively. On the other hand, the transmittance was not significantly decreased by the bending of the endo-
Table 2. Conditions for the measurement of the dependence of the output laser power and incision depth on the bending of the head of the endoscope.

\begin{tabular}{ccccc}
\hline Bending angle $\left({ }^{\circ}\right)$ & 0 & 30 & 60 & 90 \\
Bending radius $r_{2}(\mathrm{~cm})$ & - & 11.0 & 7.0 & 5.0 \\
\hline
\end{tabular}

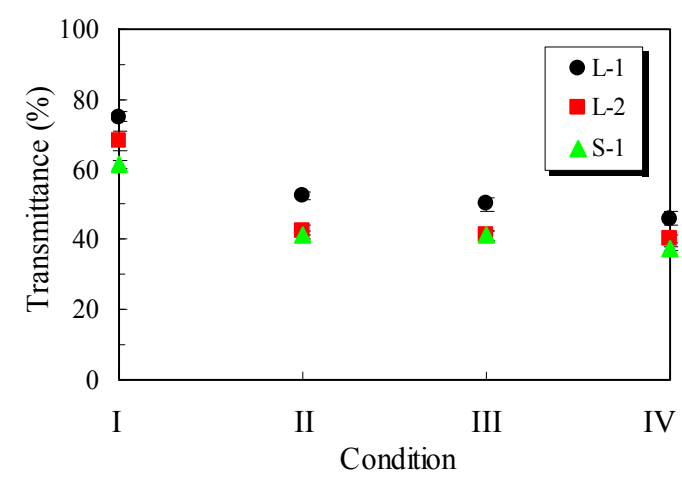

Figure 4. Transmittance of the hollow optical fibers under each condition.

scope. When the bending of the endoscope was changed from the condition II to IV, the transmittances of the fibers L-1, L-2, and S-1 decreased by 7, 3, and 4\%, respectively. Therefore, it was found that the decrease in the transmittance of the hollow optical fiber due to the insertion into the instrument channel was the most dominant loss in the hollow optical fibers. It is suggested that these results are caused by the steep bending of $130^{\circ}$ just after the insertion into the instrument channel as shown in Figure 2.

\subsection{Dependence of the Output Laser Power and Incision Depth on the Bending of the Head of the Endoscope}

Figure 5 shows the relationships between the bending angle of the tip of the hollow optical fiber and the output laser power. The output laser power gradually decreased with the increase in the bending angle of the tip of the hollow optical fiber, and the output laser power at $90^{\circ}$ was about $10 \%$ lower than that for $0^{\circ}$.

Figure 6 shows the photomicrographs of the cross sections of the HE stained porcine stomach samples after laser irradiation with each condition. Figure 7 shows the relationships between the bending angle of the tip of the hollow optical fiber and the incision depth measured from the photomicrographs of the sample shown in Figure 6. The incision depth under each condition decreased by up to $25 \%$ compared to that for $0^{\circ}$ with an exception for the condition of $8 \mathrm{~W}$ at $90^{\circ}$. Therefore, it was confirmed that the bending loss of the hollow optical fiber due to the bending of the head of the endoscope had no significant influence on the endoscopic therapy using the carbon dioxide laser. 

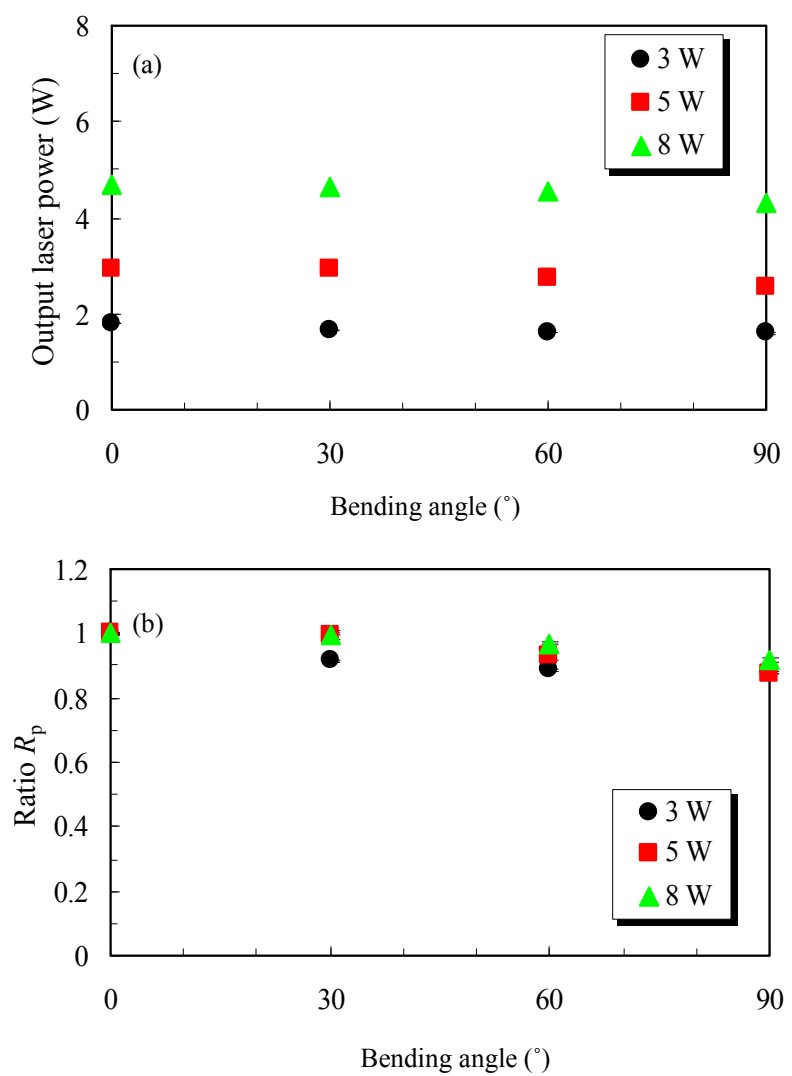

Figure 5. The relationships between (a) the bending angle of the head of the endoscope and the output laser power and (b) the bending angle and the ratio $R_{p}$ of the output laser power to that for $0^{\circ}$.

The mechanism of the vaporization of the biological soft tissue by a laser knife is described as follows: When the biological soft tissue absorbs the laser, the temperature of the tissue quickly rises within a time of microseconds. Since the boiling point of water is $100^{\circ} \mathrm{C}$, water in tissue is immadiately vaporized and the volume is expanded by continuously making water in the tissue over $100^{\circ} \mathrm{C}$. Then, cell membrane and organelle fragment are vaporized. In our experiment, laser was linerly scanned with a speed of $1.0 \mathrm{~mm} / \mathrm{s}$, incision was performed using the vaporization. In order to describe the reltionship between the irradiated laser energy density and the incision depth, the steady-state model has been proposed [8,9]. This model was developed for continuous ablation process using a continuous wave or pulsed laser with microseconds or longer pulse width. In the steady-state model, the relational expression between the incision depth $\delta$ $(\mathrm{mm})$ and the irradiated laser energy density $\Phi_{0} \quad\left(\mathrm{~J} / \mathrm{mm}^{2}\right)$ is given by

$$
\delta=\frac{\Phi_{0}-\Phi_{\mathrm{th}}}{\rho h_{\mathrm{abl}}},
$$

where $\rho=1.02 \times 10^{-3} \mathrm{~g} / \mathrm{mm}^{3}$ is the density of biological

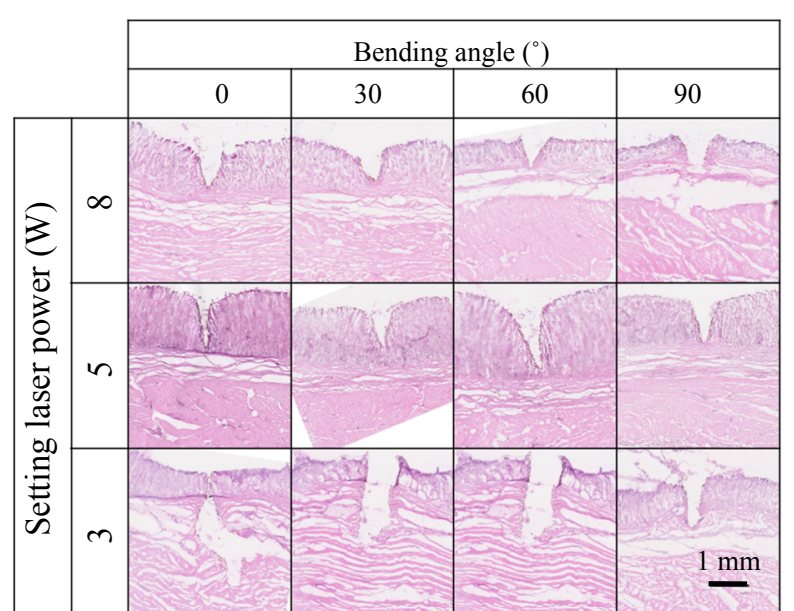

Figure 6. Photomicrographs of the HE stained samples after laser irradiation with each condition.

soft tissue composed of water of $70 \%$, and the ablation enthalpy $h_{\mathrm{abl}}(\mathrm{J} / \mathrm{g})$ and the ablation threshold energy density $\Phi_{\mathrm{th}}\left(\mathrm{J} / \mathrm{mm}^{2}\right)$ are defined as follows:

$$
\begin{gathered}
h_{\mathrm{abl}}=C \Delta T+L, \\
\Phi_{\mathrm{th}}=\frac{\rho h_{\mathrm{abl}}}{\mu_{\mathrm{a}}},
\end{gathered}
$$

where $C=3.45 \mathrm{~J} /(\mathrm{g} \cdot \mathrm{K})$ is the heat capacity, $\Delta T=63.0$ $\mathrm{K}$ is the temperature rise to the boiling point of water, $L$ $=2.58 \times 10^{3} \mathrm{~J} / \mathrm{g}$ is the heat of vaporization, and $\mu_{\mathrm{a}}=$ $6.15 \times 10^{2} \mathrm{~mm}^{-1}$ is the absorption coefficient of the porcine mucosa at the wavelength of $10.6 \mu \mathrm{m}$ [10-12]. In Figure 8, the dots show the relationship between the irradiated laser energy density and the incision depth obtained from the results in Figures 5 and 7, and the solid line represents the theoretical values estimated using Equation (1). The laser beam diameter at the surface of the porcine stomach mucosa was measured for the fiber L-1 by using knife-edge method and was about 710,712 , 772 , and $740 \mu \mathrm{m}$ at the bending angle of $0^{\circ}, 30^{\circ}, 60^{\circ}$, and $90^{\circ}$, respectively. The laser beam diameter was increased with the increase in the bending angle, because the beam spread angle from the exit of the hollow optical fiber was increased with the increase in the bending angle due to the conversion of the propagation mode from the loworder modes to the high-order modes in the hollow optical fiber.

From the results in Figure 8, it was found that both the experimental and theoretical values have similar correlations between the irradiated laser energy density and the incision depth. However, the theoretical values were about 1.5 times higher than the experimental values. It is inferred that the principal factor for this difference is the uncertainty in the physical values used in the model calculations, e.g., the density of biological soft tissue, ablation enthalpy, and the heat capacity. Since these values 

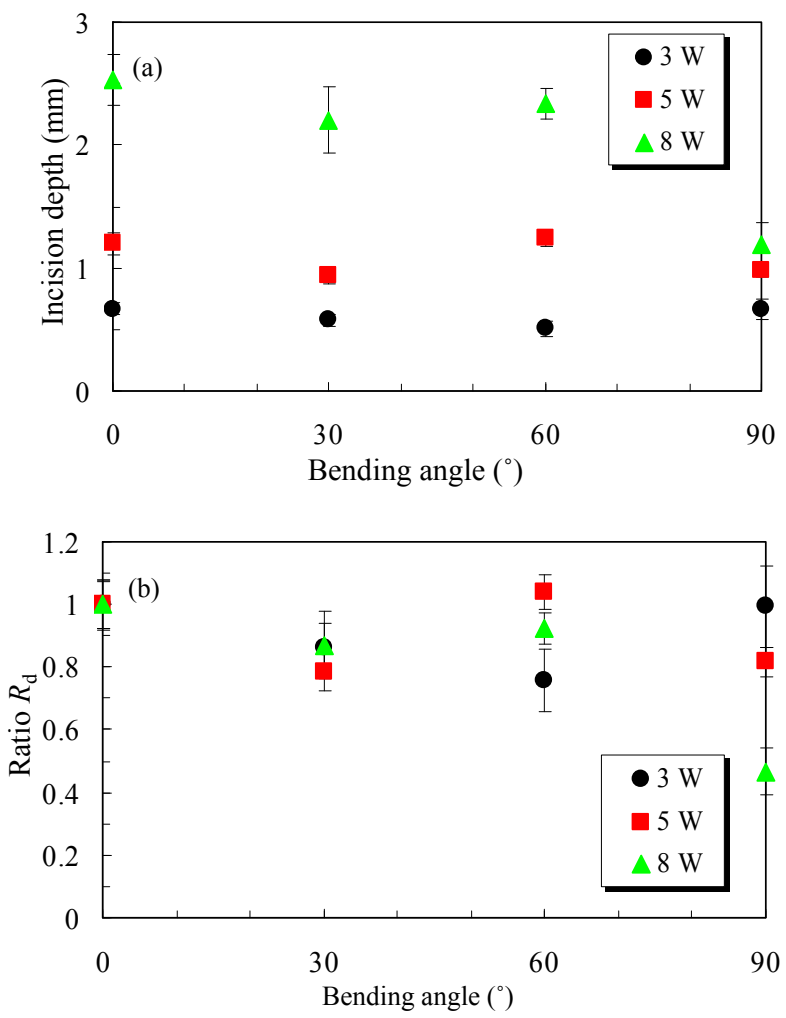

Figure 7. The relationships between (a) the bending angle of the tip of the hollow optical fiber and incision depth and (b) the bending angle and the ratio $R_{\mathrm{d}}$ of the incision depth to that for $0^{\circ}$.

were taken from the literatures, some values might not be suitable for the porcine stomach sample used in this research.

\section{Conclusion}

The change in the output power of the carbon dioxide laser and therapeutic effect by bending the hollow optical fiber inserted in a gastrointestinal endoscope was quantitatively evaluated. The change in the transmittance of the hollow optical fiber due to the insertion of the fiber to the endoscope and bending of the head of the endoscope was measured. Then, the relationship between the irradiated laser power and the incision depth of a porcine stomach was investigated. As the result, the greatest decrease of the transmittance of the hollow optical fiber was caused by insertion of the fiber into the instrument channel of the endoscope, and bending of the head of the endoscope with the angle of $90^{\circ}$ decreased the output laser power and incision depth by no more than $10 \%$ and less than $25 \%$, respectively. Therefore, it was confirmed that the bending loss of the hollow optical fiber due to the bending of the head of the endoscope had no significant influence on the endoscopic therapy using the carbon dioxide laser.

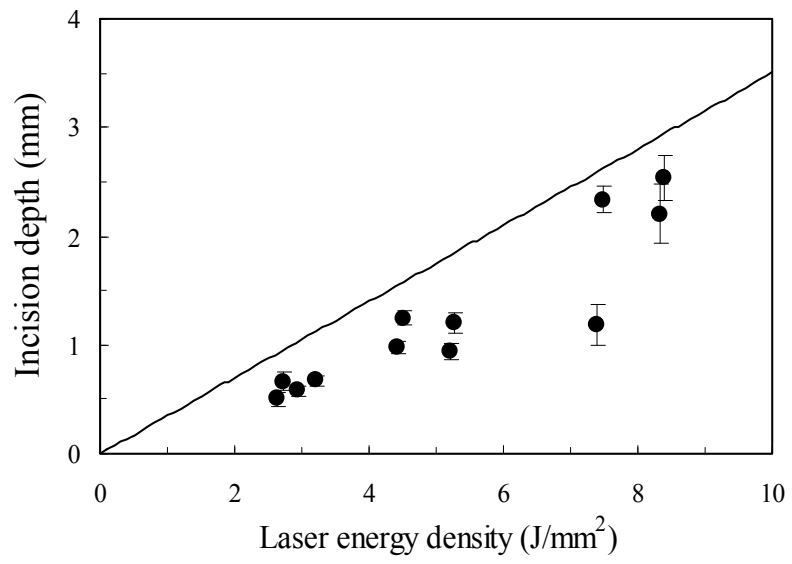

Figure 8. The relationships between the incision depth and irradiated laser energy density, where the solid line shows the theoretical values estimated with the steady-state model.

\section{Acknowledgements}

We thank Haruhiko Murakami, Katsumi Hiyoshi, Akihito Hongo, and Yoshihide Okagami of J. Morita Manufacturing Corp. for their technical supports. This work was supported by Health Labour Science Research Grant of the Ministry of Health Labour and Welfare of Japan.

\section{REFERENCES}

[1] J. T. Walsh and T. F. Deutsch, "Er:YAG Laser Ablation of Tissue: Measurement of Ablation Rates," Lasers in Surgery and Medicine, Vol. 9, No. 4, 1989, pp. 327-337. doi:10.1002/1sm.1900090404

[2] J. T. Walsh, T. J. Flotte, R. R. Anderson and T. F. Deutsch, "Pulsed $\mathrm{CO}_{2}$ Laser Tissue Ablation: Effect of Tissue Type and Pulse Duration on Thermal Damage," Lasers in Surgery and Medicine, Vol. 8, No. 2, 1988, pp. 108-118. doi:10.1002/1sm.1900080204

[3] K. Ishii, S. Watanabe, D. Obata, H. Hazama, Y. Morita, Y. Matsuoka, H. Kutsumi, T. Azuma and K. Awazu, "Selective Mucosal Ablation Using $\mathrm{CO}_{2}$ Laser for the Development of Novel Endoscopic Submucosal Dissection: Comparison of Continuous Wave and Nanosecond Pulsed Wave," Proceedings of SPIE, Vol. 7562, 2010, Article ID: 75620Q. doi: $10.1117 / 12.842426$

[4] S. R. Browd, J. Zauberman, M. Karandikar, J. G. Ojemann, A. M. Avellino and R. G. Ellenbogen, "A New Fiber-Mediated Carbon Dioxide Laser Facilitates Pediatric Spinal Cord Detethering," Journal of Neurosurgery: Pediatrics, Vol. 4, No. 3, 2009, pp. 280-284. doi:10.3171/2009.4.PEDS08349

[5] Y. Matsuura, "Basics of Optical Fibers for Medical Applications," The Journal of Japan Society for Laser Surgery and Medicine, Vol. 31, No. 4, 2011, pp. 407-412 (in Japanese).

[6] Y. Abe, Y. W. Shi, Y. Matsuura and M. Miyagi, "Flexible Small-Bore Hollow Fibers with an Inner Polymer Coating," Optics Letters, Vol. 25, No. 3, 2000, pp. 150152. doi:10.1364/OL.25.000150 
[7] Y. Matsuura, "Basics of Hollow Optical Fiber," Medical Photonics, No. 2, 2010, pp. 11-15 (in Japanese).

[8] A. Vogel and V. Venugopalan, "Mechanisms of Pulsed Laser Ablation of Biological Tissues," Chemical Reviews, Vol. 103, No. 2, 2003, pp. 577-644. doi:10.1021/cr010379n

[9] M. H. Niemz, "Laser-Tissue Interactions: Fundamentals and Applications," Springer, Berlin, 1996, pp. 58-87. doi:10.1007/978-3-662-03193-3

[10] B. Choi and A. J. Welch, "Analysis of Thermal Relaxation during Laser Irradiation of Tissue," Lasers in Surgery and Medicine, Vol. 29, No. 4, 2001, pp. 351-359. doi:10.1002/1sm.1128

[11] V. Venugopalan, N. S. Nishioka and B. B. Mikic, "Thermodynamic Response of Soft Biological Tissues to Pulsed Infrared-Laser Irradiation,” Biophysical Journal, Vol. 70, No. 6, 1996, pp. 2981-2993. doi:10.1016/S0006-3495(96)79868-5

[12] D. Obata, Y. Morita, R. Kawaguchi, K. Ishii, H. Hazama, K. Awazu, H. Kutsumi and T. Azuma, "Endoscopic Submucosal Dissection Using a Carbon Dioxide Laser with Submucosally Injected Laser Absorber Solution (Porcine Model)," Surgical Endoscopy, 2013, in Press. doi:10.1007/s00464-013-3029-x 